\title{
The effect of single trial transcutaneous electrical nerve stimulation on balance and gait function in elderly people with dementia: a pilot study
}

\author{
Ju Yeon Jung ${ }^{a, \dagger}$, Jin-Hwa Jung ${ }^{\text {b, }}{ }^{\dagger}$, Suk-Chan Hahm ${ }^{c}$, Kyoungsim Jung ${ }^{b}$, Sung-Jin Kim ${ }^{d}$, Hye Rim Suh ${ }^{\text {e, }}$, \\ Hwi-young $\mathrm{Cho}^{\mathrm{a}, *}$ \\ ${ }^{a}$ Department of Physical Therapy, College of Health Science, Gachon University, Incheon, Republic of Korea \\ bepartment of Occupational Therapy, Semyung University, Jecheon, Republic of Korea \\ 'Department of Rehabilitation Standard and Policy, National Rehabilitation Research Institute, National Rehabilitation Center, Seoul, Republic of Korea \\ ${ }^{\mathrm{d} D e p a r t m e n t ~ o f ~ P h y s i c a l ~ T h e r a p y, ~ C o l l e g e ~ o f ~ H e a l t h ~ S c i e n c e ~ a n d ~ S o c i a l ~ W e l f a r e, ~ S a h m y o o k ~ U n i v e r s i t y, ~ S e o u l, ~ R e p u b l i c ~ o f ~ K o r e a ~}$ \\ ${ }^{\mathrm{e}}$ Department of Physiology, Korea University College of Medicine, Seoul, Republic of Korea
}

Objective: Elderly people with dementia experience not only cognitive dysfunction but also motor function deficits, such as balance and gait impairments. Recently, transcutaneous electrical nerve stimulation (TENS) has been used to reduce pain as well as to control muscle spasm, spasticity and motor performance in various types of subjects. The purpose of this study determined the effect of a single trial TENS on balance and gait function in elderly people with dementia.

Design: A randomized controlled trial.

Methods: Twenty-two subjects with dementia were assigned to the TENS group $(n=12)$ and ten subjects were assigned to the control group $(n=10)$ randomly. Subjects were classified into two groups: the TENS group $(n=12)$ and the placebo-TENS group $(\mathrm{n}=10)$. The TENS group had electrical stimulation applied on the calf muscle for 15 minutes, while the placebo-TENS group had not received real electrical stimulation. The timed up and go test (TUG) and functional reach test (FRT) were used to measure the balance function, and the 10 meters walk test (MWT) and 6MWT were used to assess gait ability. All tests were performed before and after intervention under a single-blinded condition.

Results: After intervention, there were significant improvements in TUG, FRT, 10MWT, and 6MWT results in the TENS group $(p<0.05)$, while the placebo-TENS group did not show significant changes in all outcome measurements. There were also differences in all tests between the two groups at post-measurements $(p<0.05)$.

Conclusions: This study demonstrated that a single trial TENS application on the calf may be used to improve balance and gait function in elderly people with dementia.

Key Words: Balance, Dementia, Gait, Transcutaneous electric nerve stimulation

\section{Introduction}

The Korean society is moving towards an aged society.
Because of the rapidly aging society and progressively Westernized lifestyle, elderly people with dementia are increasing and have been emerged as a big social and econom-

Received: 15 May, 2017 Revised: 14 June, 2017 Accepted: 15 June, 2017

Corresponding author: Hye Rim Suh

Department of Physiology, Korea University College of Medicine, 73 Inchon-ro, Seongbuk-gu, Seoul 02841, Republic of Korea Tel: 82-2-2286-1282 Fax: 82-2-927-1036 E-mail: orange2200@hanmail.net

Corresponding author: Hwi-young Cho

Department of Physical Therapy, College of Health Science, Gachon University, 191 Hambangmoe-ro, Yeonsu-gu, Incheon 21936, Republic of Korea Tel: 82-32-820-4560 Fax: 82-32-820-4420 E-mail: hwiyoung@gachon.ac.kr

*These authors contributed equally to this work as corresponding authors.

${ }^{\dagger}$ These two authors contributed equally to this study as co-first author

(c) This is an Open-Access article distributed under the terms of the Creative Commons Attribution Non-Commercial License (http://creativecommons.org/licens es/by-nc/4.0) which permits unrestricted non-commercial use, distribution, and reproduction in any medium, provided the original work is properly cited.

Copyright $\odot 2017$ Korean Academy of Physical Therapy Rehabilitation Science 
ic problem [1]. Besides, elderly patients with dementia bring stress, depression and severe socio-economical burdens to their family and people around them [2]. Moreover, there is a high-risk of severe accidents or death for elderly people with dementia compared to ordinary elderly people due to decreased balance and gait abilities [3]. Elderly patients get hurt from a fall, which is often caused by lack of confidence. This reduces physical activity levels and decreases the quality of life [4]. To decrease the risk of falls and fall incidents of elderly patients with dementia and to increase their quality of life, interventions that increase balance and gait performance are in need. Researchers have used computer-based cognitive rehabilitational interventions [5] or nootropic drugs [6] to increase cognitive function of the patients with dementia, but the effectiveness of the treatment has not been clear.

When a treatment method was applied to family members of the patients with dementia to deal with their recent functional problems, balance was not changed while capacity for actual daily living and gait performances were increased significantly [7]. Recently, when gait training with an electrical treatment was applied to stroke patients, their balance and gait performances were increased [8]. Moreover, the strength of patients with multiple sclerosis was increased when functional electrical stimulation was applied to them [9]. According to these results, it appears that therapeutic interventions using electrical stimulation on elderly patients with dementia have an effect on their gait and balance abilities. Among many electrical treatments, transcutaneous electrical nerve stimulation (TENS) not only decreases pain according to gait control theory, but also decreases pain sensitivity by activating the descending inhibitory system [10]. Both sides of the anterior cingulated cortex of the ipsilateral supplementary motor area are activated by activating the parietal lobe of the cerebrum and the first and second somatic sensory cortices when TENS is applied [11]. When TENS is applied on the peripheral region, not only are the sensory nerves affected, but motor function can also be positively affected by activating the central nervous system. Furthermore, recent studies show that TENS is effective for not only pain reduction, but also may have many effects in improving movements. Imbalance due to fatigue is improved when TENS is applied to healthy adults [12] and studies showing that TENS is effective for balance and gait function improvement on subjects have increased [13]. However, there are only a few studies about the effect of TENS on elderly patients with dementia. Thus, this study aimed to investigate the effects of applying TENS on calf muscles in elderly people with dementia on balance and gait based on previous studies about the effect of TENS and extended usage of TENS.

\section{Methods}

\section{Subjects}

The study was conducted for 4 months as a controlled before-after pilot study. Thirty subjects that were from community centers and dementia centers in Seoul and Incheon were recruited under the agreement of the caregivers and staff. Among the 30 participants, only 25 of them had met the criteria. The subjects were randomly assigned to either the TENS group or the control group. Thirteen subjects were assigned to the TENS group and the rest of the participants were assigned to the placebo-TENS group. During the experiment, one subject from the TENS group and two from the placebo-TENS group were excluded due to refusal or unintended behavior. Thus, 12 participants in the TENS group and 10 subjects in the controlled group participated the experiment. The subjects who had mild dementia, had a score of 4 or above in the Korean mini mental state examination and were able to walk longer than 10 meter were selected. Also, the patients with other diseases such as cancer, severe cardiopulmonary symptoms, musculoskeletal problems, or received artificial joint replacements were excluded to minimize variability. The present study was approved by the Institutional Review Board of Gachon University (IRB No. 1044396-201511-HR-053-003).

\section{Procedures}

The study assigned 22 subjects with dementia to the TENS group $(n=12)$ and control group $(n=10)$ randomly. The researchers performed the evaluations after explaining or demonstrating to the subjects the evaluations 1 to 2 times. The subjects received assistance from their family or caregivers in understanding the evaluation procedures. There were 3-minutes rest periods between the evaluations to minimize fatigue after each evaluation. Every evaluation was conducted by blinded examiners. The TENS parameters were a frequency of $4 \mathrm{~Hz}$ and duration of $200 \mu \mathrm{s}$ and set at burst mode for 15 minutes on both calf muscles to provoke muscle contraction. The placebo-TENS was applied as the same as the TENS group without stimulation. The timed up and go test (TUG), functional reach test (FRT), 10 meters walk test (MWT), and 6MWT scores were used to measure 
balance and gait performance of the elderly patients with dementia. The measurements were performed before and right after the intervention.

\section{Outcome measurements}

\section{Balance measurements}

The TUG was used to measure functional mobility. The TUG is a quick method used to measure basic mobility and balance. It measures the distance from sitting on a chair with an armrest to standing, and then walking a 3 meters distance after the start sign has been initiated by the inspector. Testretest reliability of the TUG was reported as intra-class correlation coefficient $=0.96$ [14]. The less time it takes for a subject to perform the TUG indicates that the subject has good balance. The FRT has the subject stand right beside the wall until one's shoulder touches the wall and has to raise the right shoulder to 90 degrees and after leaning forward as possible as can distance from baseline is measured at the third metacarpal bone [15]. The FRT is precise, stable, is associated with the center of pressure excursion and is reported to have a reliability of $r=0.71-0.81$ [16]. Every test was performed three times with the mean values obtained.

\section{Gait measurements}

The 10MWT and 6MWT were used to measure gait. The 10MWT calculates subject's speed by measuring the time that one can walk as fast as possible. The subjects walked 4 meters more before and after the starting and the 10 meters mark to exclude the effects of acceleration and deceleration when measuring the time it took for the subject to walk 10 meters [17]. This tool has a high reliability of $r=0.89-1.00$ ) [18] and every test used the mean values obtained from three trials. The method of 6MWT was the same as 10MWT, but the length was set at 6 meters only. The method showed an inter-rater reliability of 0.88 and an intra-rater reliability of

Table 1. General characteristics of participants $(\mathrm{N}=22)$

\begin{tabular}{lccc}
\hline \multicolumn{1}{c}{ Characteristic } & $\begin{array}{c}\text { TENS group } \\
(\mathrm{n}=12)\end{array}$ & $\begin{array}{c}\text { Placebo-TENS } \\
\text { group }(\mathrm{n}=10)\end{array}$ & $p$ \\
\hline Age $(\mathrm{y})$ & $79.03(5.67)$ & $78.33(9.32)$ & 0.830 \\
Sex (male/female) & $6 / 6$ & $4 / 6$ & 0.658 \\
Height (cm) & $165.42(8.23)$ & $162.78(6.85)$ & 0.823 \\
Weight $(\mathrm{kg})$ & $62.49(10.22)$ & $61.69(9.07)$ & 0.904 \\
K-MMSE & $17.03(5.24)$ & $16.91(6.83)$ & 0.820 \\
\hline
\end{tabular}

Values are presented as mean (SD).

TENS: transcutaneous electrical nerve stimulation, K-MMSE: Korean mini mental state examination.
0.86 when elderly people with dementia were measured and was considered to be reliable when applied to them [19].

\section{Statistical analysis}

General characteristics were analyzed by frequency analysis using IBM SPSS Statistics ver. 23.0 (IBM Co., Armonk, NY, USA) and test of normality was done by the Shapirowilk test. An independent t-test was used to compare the changes of pre- and post-test and the paired t-test was used to compare before and after intervention. The significance level was set lower than 0.5 .

\section{Results}

\section{Demographic characteristics}

There was no significant difference in general characteristics of subjects such as sex, age, height, and weight for dementia between the groups ( $p>0.05$; Table 1$)$.

\section{Balance}

In the TENS group, there was a significant difference in TUG scores from $13.48 \pm 4.28$ seconds before the intervention to $11.94 \pm 3.41$ seconds after the intervention $(p<$ $0.05)$. Meanwhile, there was no significant difference in the placebo-TENS group. In comparison of the changes, the TENS group showed significant improvement compared to the placebo-TENS group ( $p<0.05$; Table 2 ).

In FRT test, while the TENS group showed significant difference of a total change of $1.92 \pm 2.50 \mathrm{~cm}$ from $17.87 \pm$ $6.66 \mathrm{~cm}$ before the intervention to $19.79 \pm 7.37 \mathrm{~cm}$ after the intervention $1.92 \pm 2.50 \mathrm{~cm}(p<0.05)$, the placebo-TENS

Table 2. The changes in TUG and FRT scores

$(\mathrm{N}=22)$

\begin{tabular}{cccc}
\hline Variable & $\begin{array}{c}\text { TENS group } \\
(\mathrm{n}=12)\end{array}$ & $\begin{array}{c}\text { Placebo-TENS } \\
\text { group }(\mathrm{n}=10)\end{array}$ & $p$ \\
\hline TUG $(\mathrm{s})$ & & & \\
Pre-test & $13.48(4.28)$ & $12.04(4.11)$ & 0.434 \\
Post-test & $11.94(3.41)$ & $12.08(3.88)$ & \\
Post-Pre & $1.54(1.94)$ & $0.04(0.65)$ & 0.019 \\
$p$ & 0.016 & 0.850 & \\
FRT $(\mathrm{cm})$ & & & \\
Pre-test & $17.87(6.66)$ & $20.07(3.76)$ & 0.383 \\
Post-test & $19.79(7.37)$ & $18.65(4.43)$ & \\
Post-Pre & $1.92(2.50)$ & $1.42(2.52)$ & 0.007 \\
$p$ & 0.026 & 0.123 & \\
\hline
\end{tabular}

Values are presented as mean (SD).

TUG: timed up and go test, FRT: functional reach test, TENS: transcutaneous electrical nerve stimulation. 
Table 3. The changes of the 10MWT and 6MWT scores $(\mathrm{N}=22)$

\begin{tabular}{cccc}
\hline Variable & $\begin{array}{c}\text { TENS group } \\
(\mathrm{n}=12)\end{array}$ & $\begin{array}{c}\text { Placebo-TENS } \\
\text { group }(\mathrm{n}=10)\end{array}$ & $p$ \\
\hline 10MWT (s) & & & \\
Pre-test & $11.43(3.20)$ & $10.34(3.07)$ & 0.399 \\
Post-test & $9.71(2.52)$ & $10.83(3.02)$ & \\
Post-Pre & $1.72(1.24)$ & $0.49(1.50)$ & 0.001 \\
$p$ & 0.001 & 0.281 & \\
6MWT (s) & & & \\
Pre-test & $7.58(2.00)$ & $6.93(2.26)$ & 0.479 \\
Post-test & $6.42(1.70)$ & $6.76(2.37)$ & \\
Post-Pre & $1.16(0.58)$ & $0.17(0.52)$ & $<0.001$ \\
$p$ & $<0.001$ & 0.390 & \\
\hline
\end{tabular}

Values are presented as mean (SD).

MWT: meter walk test, TENS: transcutaneous electrical nerve stimulation.

group showed a decline from $20.07 \pm 3.76 \mathrm{~cm}$ before the intervention to $18.65 \pm 4.43 \mathrm{~cm}$ after the intervention, although the decline was not significant $(p>0.05)$. In comparison of the changes, the TENS group showed significant improvement compared to the placebo-TENS group $(p<0.05$; Table 2).

\section{Gait}

In the 10MWT test, while the TENS group showed significant improvement of approximately 1.7 seconds from $11.43 \pm 3.20$ seconds before the intervention to $9.71 \pm 2.52$ seconds after the intervention $(p<0.05)$, the placebo-TENS group showed no significant difference from $10.34 \pm 3.07$ seconds before the intervention to $10.83 \pm 3.02$ seconds after the intervention $(p>0.05)$. The changes in the TENS group showed a significant improvement in gait function compared to the placebo-TENS group ( $p<0.05$; Table 3$)$. Moreover, there was a similar change in the 6MWT scores as for the 10MWT. The TENS group had shown significant improvement $(p<0.05)$, but the placebo-TENS group showed no significant difference $(p>0.05)$. The changes of the TENS group showed significant improvements in gait function compared to the placebo-TENS group $(p<0.05$; Table 3$)$.

\section{Discussion}

Gait and balance functions of elderly patients with dementia are decreased due to reduction of cognition and muscle weakness. Decrease of gait speed is a leading index of gait disorders of patients with dementia and was reported to be highly correlated with executive function disorder [20].
The study proved that the application of TENS improved the gait speeds of elderly patients with dementia. The results of this study were similar to previous studiesin which dynamic balance, such as gait, was improved when active-TENS was applied to stroke patients [21]. Balance was improved in the TENS group as demonstrated by the TUG and FRT tests. This appears to be because TENS increased the activation of the somatic sensory system that was engaged in the maintenance and control of the standing position [22]. Similar to the previous studies, TENS on stroke patients showed that balance and gait performance was improved [21] as well as standing balance because perturbation was decreased when TENS was applied to young healthy adults [23]. Balance improvement allows elderly people with dementia to be able to perform ADLs and live an independent life safely by decreasing the risk of falls [24]. Meanwhile, there was no significant improvement in not only the 10MWT, but also in TUG and FRT scores in the placebo-TENS group. This clearly means that TENS without electronic charge does not have much of an effect on patients with dementia.

Apparently, elderly people with dementia avoid activities because they are hypoactive. This leads to muscle weakness and decreased muscular activation. It is assumed that applying TENS increases gait performance in the study with a few reasons. First of all, the electrical stimulation on the peripheral nerves activates the brain's motor area. According to previous studies, cerebral activity was activated due to electrical stimulation on the peripheral nerves [25]. Second, it is assumed that the muscular activation of elderly patients was increased because TENS stimulation on the calf muscle produced repeated muscle contractions passively. Based on the previous studies, applying TENS increases muscular activation and strength, and therefore, motor function related with them is also increased [26]. TENS activates slow-twitch and fast-twitch muscles fibers, such as the Ia and II fibers [27]. Applying TENS increased muscle activation through repeated passive muscular contractions, which overcame the limitation of lack of activity many elderly patient with dementia suffer from. Lastly, continuous stimulation on the nerves may have activated the motor and sensory integration area.

The 10MWT and the 6MWT were used to measure gait performance in the study. These two methods are the same except the distance of 10 meters and 6 meters. The 6MWT had used to measure gait performance of elderly people with dementia while the 10MWT had been used in several studies about neurological deficits [19]. It seems that these methods reduce the burdens associated with measuring elderly peo- 
ple with dementia, and are effective. In the results of the study, both methods showed significant improvement in the TENS group. However, there was, interestingly, a difference in improvement between the 6MWT and the 10MWT. The subjects were enhanced by $15.8 \%$ in the $6 \mathrm{MWT}$ and $22 \%$ in the 10MWT. It is assumed that the 10MWT is more effective for measuring gait performance of the elderly with dementia even though it is difficult to be generalized due to the small number of subjects. Long distances require more time to react after by patients compared to short distances. Nevertheless, it is assumed that both methods are effective for measuring gait performances of the elderly with dementia and there will be studies comparing the advantages and disadvantages of them in the future.

This study has some limitations. First, it is difficult to ascertain long-term effect of TENS because TENS was applied one time on each subject. Also, it is difficult to know how long the effects had been sustained after the intervention due to no follow-up study. Thus, further studies need to investigate the effect of applying TENS for an extended period of time and to ascertain how long effects have been sustained in the future and neurological-physiological studies about how TENS affects gait, balance, and strength and pain sensory should be warranted as well.

The study proved that applying TENS on the elderly with dementia is effective for improvement of balance and gait performance. It is assumed that using TENS in the field improves motor function as well as reduces pain through the study result. There is advantages, especially, that TENS is easy to use because it is not invasive and safe and few disadvantages. Furthermore, it also induces continuous muscle contraction passively. Which make the elderly with dementia overcome hypoactivity. Thus, TENS is considered as an useful equipment to improve motor function of elderly patients with dementia.

\section{Acknowledgements}

This research was supported by Creative Korea II Program (CK-II) through the National Research Foundation of Korea (NRF) funded by the Ministry of Education (NRF-2014 H1C9A2A02024284).

\section{Conflict of Interest}

The authors declared no potential conflicts of interest with respect to the authorship and/or publication of this article.

\section{References}

1. Lee S, Kang H, Shin G. Head flexion angle while using a smartphone. Ergonomics 2015;58:220-6.

2. Xie Y, Szeto GP, Dai J, Madeleine P. A comparison of muscle activity in using touchscreen smartphone among young people with and without chronic neck-shoulder pain. Ergonomics 2016; 59:61-72.

3. Lauche R, Cramer H, Choi KE, Rampp T, Saha FJ, Dobos GJ, et al. The influence of a series of five dry cupping treatments on pain and mechanical thresholds in patients with chronic non-specific neck pain--a randomised controlled pilot study. BMC Complement Altern Med 2011;11:63.

4. Christie HJ, Kumar S, Warren SA. Postural aberrations in low back pain. Arch Phys Med Rehabil 1995;76:218-24.

5. Gelb DE, Lenke LG, Bridwell KH, Blanke K, McEnery KW. An analysis of sagittal spinal alignment in 100 asymptomatic middle and older aged volunteers. Spine (Phila Pa 1976) 1995;20:1351-8.

6. Straker LM, Coleman J, Skoss R, Maslen BA, Burgess-Limerick $\mathrm{R}$, Pollock CM. A comparison of posture and muscle activity during tablet computer, desktop computer and paper use by young children. Ergonomics 2008;51:540-55.

7. Krauss J, Creighton D, Ely JD, Podlewska-Ely J. The immediate effects of upper thoracic translatoric spinal manipulation on cervical pain and range of motion: a randomized clinical trial. J Man Manip Ther 2008;16:93-9.

8. Tousignant M, de Bellefeuille L, O'Donoughue S, Grahovac S. Criterion validity of the cervical range of motion (CROM) goniometer for cervical flexion and extension. Spine (Phila Pa 1976) 2000;25:324-30.

9. Rainville J, Hartigan C, Martinez E, Limke J, Jouve C, Finno M. Exercise as a treatment for chronic low back pain. Spine J 2004; 4:106-15.

10. Chi LM, Lin LM, Chen CL, Wang SF, Lai HL, Peng TC. The effectiveness of cupping therapy on relieving chronic neck and shoulder pain: a randomized controlled trial. Evid Based Complement Alternat Med 2016;2016:7358918.

11. Clare HA, Adams R, Maher CG. Reliability of McKenzie classification of patients with cervical or lumbar pain. J Manipulative Physiol Ther 2005;28:122-7.

12. Quek J, Brauer SG, Treleaven J, Pua YH, Mentiplay B, Clark RA. Validity and intra-rater reliability of an android phone application to measure cervical range-of-motion. J Neuroeng Rehabil 2014;11:65.

13. de Koning CH, van den Heuvel SP, Staal JB, Smits-Engelsman $\mathrm{BC}$, Hendriks EJ. Clinimetric evaluation of active range of motion measures in patients with non-specific neck pain: a systematic review. Eur Spine J 2008;17:905-21.

14. McKenzie R. The cervical and thoracic spine: mechanical diagnosis and therapy. Waikanae, New Zealand: Spinal Publications (N.Z.) Ltd.; 1990.

15. Grace EG, Sarlani E, Reid B. The use of an oral exercise device in the treatment of muscular TMD. Cranio 2002;20:204-8.

16. Duncan PW, Weiner DK, Chandler J, Studenski S. Functional reach: a new clinical measure of balance. J Gerontol 1990;45: 
M192-7.

17. Vikne J, Oedegaard A, Laerum E, Ihlebaek C, Kirkesola G. A randomized study of new sling exercise treatment vs traditional physiotherapy for patients with chronic whiplash-associated disorders with unsettled compensation claims. J Rehabil Med 2007; 39:252-9.

18. Puentedura EJ, Huijbregts PA, Celeste S, Edwards D, In A, Landers MR, et al. Immediate effects of quantified hamstring stretching: hold-relax proprioceptive neuromuscular facilitation versus static stretching. Phys Ther Sport 2011;12:122-6.

19. Loew M, Heichel TO, Lehner B. Intraarticular lesions in primary frozen shoulder after manipulation under general anesthesia. J Shoulder Elbow Surg 2005;14:16-21.

20. Kim SJ. Influences of cryotherapy and intermittent compression on experimental delayed onset muscle soreness [Master thesis]. Daegu: Daegu University; 2001.

21. Schulte E. Acupuncture: where east meets west. RN 1996;59: 55-7.
22. Torebjörk E. Nociceptor activation and pain. Philos Trans R Soc Lond B Biol Sci 1985;308:227-34.

23. Michalsen A, Bock S, Lüdtke R, Rampp T, Baecker M, Bachmann $\mathrm{J}$, et al. Effects of traditional cupping therapy in patients with carpal tunnel syndrome: a randomized controlled trial. J Pain 2009;10:601-8.

24. Kaptchuk TJ, Stason WB, Davis RB, Legedza AR, Schnyer RN, Kerr CE, et al. Sham device v inert pill: randomised controlled trial of two placebo treatments. BMJ 2006;332:391-7.

25. Kim EJ, Kim JW, Park BR. Effects of sling exercise program on muscle activity and cervical spine curvature of forward head posture. J Digit Contents Soc 2011;11:213-20.

26. Sohn JH, Choi HC, Lee SM, Jun AY. Differences in cervical musculoskeletal impairment between episodic and chronic tension-type headache. Cephalalgia 2010;30:1514-23.

27. Musial F, Spohn D, Rolke R. Naturopathic reflex therapies for the treatment of chronic back and neck pain-part 1: neurobiological foundations. Forsch Komplementmed 2013;20:219-24. 